Article

\title{
Vaccination with Schistosoma mansoni Cholinesterases Reduces the Parasite Burden and Egg Viability in a Mouse Model of Schistosomiasis
}

\author{
Bemnet A. Tedla, Darren Pickering, Luke Becker, Alex Loukas and Mark S. Pearson * \\ Centre for Molecular Therapeutics, Australian Institute of Tropical Health and Medicine, James Cook University, \\ Cairns, QLD 4878, Australia; amarebem6@gmail.com (B.A.T.); darren.pickering@jcu.edu.au (D.P.); \\ luke.becker@jcu.edu.au (L.B.); alex.loukas@jcu.edu.au (A.L.) \\ * Correspondence: mark.pearson@jcu.edu.au; Tel.: +61-7-42321865
}

Received: 3 March 2020; Accepted: 1 April 2020; Published: 3 April 2020

\begin{abstract}
Schistosomiasis is a neglected tropical disease caused by parasitic blood flukes of the genus Schistosoma, which kills 300,000 people every year in developing countries, and there is no vaccine. Recently, we have shown that cholinesterases (ChEs) — enzymes that regulate neurotransmission-from Schistosoma mansoni are expressed on the outer tegument surface and present in the excretory/secretory products of larval schistosomula and adult worms, and are essential for parasite survival in the definitive host, highlighting their utility as potential schistosomiasis vaccine targets. When treated in vitro with anti-schistosome cholinesterase ( $\mathrm{SmChE}$ ) IgG, both schistosomula and adult worms displayed significantly decreased ChE activity, which eventually resulted in parasite death. Vaccination with individual $S m \mathrm{ChEs}$, or a combination of all three $S m \mathrm{ChEs}$, significantly reduced worm burdens in two independent trials compared to controls. Average adult worm numbers and liver egg burdens were significantly decreased for all vaccinated mice across both trials, with values of $29-39 \%$ and $13-46 \%$, respectively, except for those vaccinated with SmAChE1 in trial 1. Egg viability, as determined by egg hatching from liver homogenates, was significantly reduced in the groups vaccinated with the SmChE cocktail (40\%) and SmAChE2 (46\%). Furthermore, surviving worms from each vaccinated group were significantly stunted and depleted of glycogen stores, compared to controls. These results suggest that $S m \mathrm{ChEs}$ could be incorporated into a vaccine against schistosomiasis to reduce the pathology and transmission of this debilitating disease.
\end{abstract}

Keywords: schistosomiasis; vaccine; cholinesterase

\section{Introduction}

Schistosomiasis is caused by an infection with parasitic blood flukes of the genus Schistosoma, killing 300,000 people every year and infecting hundreds of millions more in developing countries [1]. The disease is spread when humans come into contact with the water-borne, infective stage of the parasite- the cercariae-which penetrate the skin and migrate through the vasculature and lungs before entering the venous system, where they become sexually mature adults that pair and mate. Eggs laid by the female are then passed in the urine or feces to the environment, which continues the transmission of schistosomiasis [1]. Eggs are deposited in tissues and organs of the host, such as the liver, and much of the disease pathology is a product of the immune response against these trapped ova, where the ensuing granulomatous lesions lead to fibrosis, which can cause severe circulatory impairment of the affected organs [2].

Despite decades of concentrated research, there is still no effective and practical vaccine against the disease [3]. Furthermore, mass chemotherapy using praziquantel (PZQ)—the only effective 
anti-schistosomal drug - is complicated by rapid and frequent reinfection [4]. There is also evidence suggesting that PZQ may be having a reduced efficacy in the treatment of infection [5].

So far, a considerable number of schistosome antigens have been identified and tested as vaccines and, although a number of these vaccine candidates (for example, SmTSP-2, Sm14, Sm29, SmCB1, and $S m p 80$ ) have shown promising efficacy in animal models and are in various stages of pre-clinical or clinical development, none have been approved for licensure [reviewed in [3]].

Due to the fundamental roles they play in parasite biology (reviewed in [6]), schistosome cholinesterases ( $\mathrm{SmChEs}$ ) have been posited as intervention targets against schistosomiasis and there are several indications to support the feasibility of their use as vaccines. Firstly, $S m C h E s$ have been localized to the tegument (outer, host-exposed surface) of schistosomula and adult worms $[7,8]$ and anti-Sm $\mathrm{ChE}$ antibodies have been shown to bind to and kill schistosomula [9], suggesting that the enzymes are accessible to immune attack. Anti-Sm $\mathrm{ChE}$ antibodies also showed no cross-reactivity against human acetylcholinesterase (AChE) [7], indicating that a vaccine safe for human use could be designed. Thirdly, protein array studies have detected significantly high levels of antibodies to SmChEs in humans exhibiting a resistance and low pathology to schistosomiasis, suggesting an involvement of these antibodies in a protective anti-schistosomal response $[10,11]$. Most of these studies have employed the use of anti-SmChE antibodies raised against biochemically purified material, so the vaccine efficacy of any one $S m \mathrm{ChE}$ paralog remains to be elucidated.

A recent study by us [8] documented the existence of three $S m C h E$ paralogs (two acetylcholinesterases—smache1 and smache — and one butyrylcholinesterase (BChE) — smbche1), and we showed that each molecule localized to the tegument of adults and schistosomula and demonstrated, through RNAi-mediated suppression in vitro and in vivo, that each paralog was essential to parasite survival. We also reported a significant reduction in the glucose-scavenging ability of silenced parasites, providing evidence for the involvement of tegumental $\mathrm{AChE}$ in the mediation of exogenous glucose uptake, which has also been documented by other studies [12-14]. Despite the fundamental roles that SmChEs appear to play in parasitism, it remains to be determined which $S m C h E$ paralogs are effective vaccine targets in Schistosoma mansoni.

Herein, we demonstrate that purified IgG against each of the three SmChE paralogs inhibits ChE activity in both larval and adult worms in vitro, which results in eventual parasite death. Furthermore, we document the efficacy of these $S m C h E s$, when administered as recombinant vaccines in isolation or as a triple combination, in reducing the parasite burden, stunting worm growth, and decreasing egg viability.

\section{Materials and Methods}

\subsection{Ethics Statement}

All experimental procedures reported in the study were approved by the James Cook University (JCU) animal ethics (Ethics approval numbers A2391). Mice were maintained in cages in the university's quarantine facility (Q2152) for the duration of the experiments. The study protocols were in accordance with the 2007 Australian Code of Practice for the Care and Use of Animals for Scientific Purposes and the 2001 Queensland Animal Care and Protection Act.

\subsection{Parasites}

Biomphalaria glabrata snails infected with S. mansoni (NMRI strain) were obtained from the Biomedical Research Institute (BRI) (MD, USA). Cercariae were shed by exposure to light at $28^{\circ} \mathrm{C}$ for $1.5 \mathrm{~h}$ and mechanically transformed to obtain schistosomula [15]. To obtain adult worms, 6-8-week-old male BALB/c mice (Animal Resource Centre, WA) were infected with 120 cercariae via tail penetration and parasites harvested by vascular perfusion at 7-8 weeks post-infection [16]. 


\subsection{Recombinant Protein Expression and Purification}

Complete open reading frames (ORFs) for smache1, smbche1, and smache2 were synthesized by Genewiz. Attempts to express full-length sequences in E. coli were unsuccessful, so primer sets incorporating $\mathrm{NdeI}$ (forward primer) and XhoI restriction enzyme sites (reverse primer) were designed to amplify partial, non-conserved regions of each smche [8], which might prove more amenable to expression. Sequences (containing NdeI/ XhoI sites) for each SmChE were amplified from each full-length template by a polymerase chain reaction (PCR) and cloned into the pET41a expression vector (Novagen) such that the N-terminal GST tag was removed. Protein expression was induced for $24 \mathrm{~h}$ in E. coli BL21 (DE3) by the addition of $1 \mathrm{mM}$ isopropyl beta-D-1-thiogalactopyranoside (IPTG) using standard methods. Cultures were harvested by centrifugation $(8000 \times g$ for $20 \mathrm{~min}$ at $4{ }^{\circ} \mathrm{C}$ ), re-suspended in $50 \mathrm{~mL}$ lysis buffer (50 mM sodium phosphate, pH 8.0, $300 \mathrm{mM} \mathrm{NaCl}, 40 \mathrm{mM}$ imidazole) and stored at $-80{ }^{\circ} \mathrm{C}$. Cell pellets were lysed by three freeze-thaw cycles at -80 and $42{ }^{\circ} \mathrm{C}$, followed by sonication on ice $(10 \times 5 \mathrm{~s}$ pulses [70\% amplitude] with $30 \mathrm{~s}$ rest periods between each pulse) with a Qsonica Sonicator. Triton X-100 was added to each lysate at a final concentration of 3\% and incubated for $1 \mathrm{~h}$ at $4{ }^{\circ} \mathrm{C}$ with end-over-end mixing. Insoluble material (containing $\mathrm{SmChEs)} \mathrm{was}$ pelleted by centrifugation at $20,000 \times g$ for $20 \mathrm{~min}$ at $4^{\circ} \mathrm{C}$. The supernatant was discarded, and inclusion bodies (IBs) were washed twice by resuspension in $30 \mathrm{~mL}$ of lysis buffer, followed by centrifugation at $20,000 \times g$ for $20 \mathrm{~min}$ at $4^{\circ} \mathrm{C}$. IBs were then solubilized sequentially by resuspension in $25 \mathrm{~mL}$ lysis buffers containing either 2,4 , or $8 \mathrm{M}$ urea; end-over-end mixing overnight at $4{ }^{\circ} \mathrm{C}$; and centrifugation at $20,000 \times \mathrm{g}$ for $20 \mathrm{~min}$ at $4{ }^{\circ} \mathrm{C}$. Finally, supernatant containing solubilized IBs was diluted 1:4 in lysis buffer containing $8 \mathrm{M}$ urea and filtered through a $0.22 \mu \mathrm{m}$ membrane (Millipore). Solubilized IBs were purified by immobilized metal affinity chromatography (IMAC) by loading onto a prepacked $1 \mathrm{~mL}$ His-Trap HP column (GE Healthcare) equilibrated with lysis buffer containing $8 \mathrm{M}$ urea at a flow rate of $1 \mathrm{~mL} / \mathrm{min}$ using an AKTA-pure-25 FPLC (GE Healthcare). After washing with $20 \mathrm{~mL}$ lysis buffer containing $8 \mathrm{M}$ urea, bound His-tagged proteins were eluted using the same buffer with a stepwise gradient of $50-250 \mathrm{mM}$ imidazole (50 mM steps). Fractions containing SmChEs (as determined by SDS-PAGE) were pooled and concentrated using Amicon Ultra-15 centrifugal devices with a $3 \mathrm{kDa}$ MWCO and quantified using the Pierce BCA Protein Assay kit. The final concentration of each $S m \mathrm{ChE}$ was adjusted to $1 \mathrm{mg} / \mathrm{mL}$ and proteins were aliquoted and stored at $-80^{\circ} \mathrm{C}$.

\subsection{Generation of Anti-Smche Antisera and Purification of IgG}

Three groups of five male BALB/c mice (6-week-old) were intraperitoneally immunized with either SmAChE1, SmBChE1, or SmAChE2 subunits $(50 \mu \mathrm{g} / \mathrm{mouse})$. Antigens were mixed with an equal volume of Imject alum adjuvant (Thermofisher) and administered three times, two weeks apart. Two weeks after the final immunization, mice were sacrificed and blood was collected via cardiac puncture. Blood from all mice in each group was pooled and serum was separated by centrifugation after clotting and stored at $-20^{\circ} \mathrm{C}$. Polyclonal antibodies were purified from mouse sera using Protein A Sepharose-4B (Thermofisher), according to the manufacturer's instructions. Serum from naïve mice was similarly processed.

\subsection{Effect of Polyclonal Anti-SmChE IgG on Larval Worms}

Newly transformed schistosomula (1000/mL) were cultured in Dulbecco's Modified Eagle Medium (DMEM) (supplemented with $4 \times \mathrm{AA}$ ) at $37{ }^{\circ} \mathrm{C}$ and $5 \% \mathrm{CO}_{2}$ in the presence of $50 \mu \mathrm{g}$ of either anti-SmAChE1, SmBChE1, or SmAChE2 polyclonal IgG or a combination of all three antibodies (equal amounts $-50 \mu \mathrm{g}$ total). Separate sets of parasites were similarly incubated with $50 \mu \mathrm{g}$ of naïve mouse IgG, which served as a control. After 2 and $14 \mathrm{~h}$ (separate experiments were conducted for each timepoint), 300 schistosomula from each experiment were removed and assessed for viability using Trypan Blue exclusion (100 parasites in triplicate) [17]. Surface and secreted ChE activity was measured using the remaining parasites by incubating them in $0.5 \mathrm{~mL}$ of assay buffer $(0.1 \mathrm{M}$ sodium 
phosphate, $\mathrm{pH} 7.4,2 \mathrm{mM}$ acetylthiocholine [AcSCh], or $2 \mathrm{mM}$ butyrylthiocholine [BcSCh], and $0.5 \mathrm{mM}$ 5, 5'-dithio-bis 2-nitrobenzoc acid [DTNB]) and monitoring the absorbance increase (AcSCh conversion or BcSCh conversion) over $1 \mathrm{~h}$ at $405 \mathrm{~nm}$ in a Polarstar Omega microplate reader (BMG Labtech). Parasites cultured with naïve IgG served as a negative control. Data are presented as the average of two biological and three technical replicates \pm SEM.

\subsection{Effect of Polyclonal Anti-SmChE IgG on Adult Worms}

Enzyme inhibitory effects $24 \mathrm{~h}$ after the addition of $\operatorname{IgG}$ (including naïve $\operatorname{IgG}$ ) were measured as for schistosomula using five pairs of freshly perfused adult worms in $1 \mathrm{~mL}$ of media. Data are presented as the average of two biological and two technical (four total) replicates \pm SEM. To investigate the effects of polyclonal anti-SmChE IgG on the worm viability, ten pairs of worms were similarly incubated with antibodies for 10 days, monitored every $24 \mathrm{~h}$ for motility by microscopic examination, and considered dead if no movement was seen. To measure the effect of polyclonal anti-SmChE IgG on glucose uptake, ten pairs of worms were similarly incubated with antibodies for $24 \mathrm{~h}$ and transferred to DMEM $(1000 \mathrm{mg} / \mathrm{L})$ and then the media glucose concentration was measured over a $24 \mathrm{~h}$ period using a glucose assay kit (Sigma). Glucose levels were expressed relative to media collected from worms which received naïve IgG (negative control). Data are presented as the average of two biological replicates \pm SEM.

\subsection{Anti-SmChE IgG Responses in S. mansoni-Infected Mice during Infection and Before and After PZQ Treatment}

Sera from S. mansoni-infected male BALB/c mice (6-8 weeks) $(n=5)$ were collected at day 3, 14, 28, 42 , and 56 post infection (p.i.) to assess anti-Sm $\mathrm{ChE}$ responses during the course of parasite infection. In a separate experiment, sera from $S$. mansoni-infected male BALB/c mice (6-8 weeks) $(n=11)$ were collected at 5 weeks p.i. and mice were then treated orally with PZQ $(100 \mathrm{mg} / \mathrm{kg})$ at 35, 37, and 39 days p.i. Sera were again collected at day 49 p.i. (2 weeks post-PZQ treatment). Anti-SmChE responses during infection and before and after PZQ treatment were screened by ELISA with plated SmChEs (100 ng/well) using standard methods. The cutoff value for each dilution was established as three times the mean OD of the naïve sera for that dilution and the endpoint was defined as the highest dilution above the cutoff value.

\subsection{Vaccine Trials}

Five groups of 10 male BALB/c mice (6-8 weeks) were immunized intraperitoneally on day $1(50 \mu \mathrm{g} /$ mouse) with either SmAChE1, SmBChE1, SmAChE2, a combination of all three SmChEs (17 $\mu \mathrm{g}$ each $-50 \mu \mathrm{g}$ total), or PBS, each formulated with an equal volume of Imject alum adjuvant (Thermofisher) and $5 \mu \mathrm{g}$ of CpG ODN1826 (InvivoGen). Immunizations were repeated on day 15 and 29 and each mouse was infected by tail penetration with $120 \mathrm{~S}$. mansoni cercariae [18] on day 43. Two independent trials were performed to ensure reproducibility. Blood was sampled at day 28 and 42 and on the day of a necropsy, to determine pre- and post-challenge antibody titers.

\subsection{Mouse Necropsy and Estimation of Worm and Egg Burden}

Mice were necropsied at day 91 (7 weeks p.i.) and worms were harvested by vascular perfusion and counted. Worms from the mice in each group were pooled and a random sample of each pool was photographed and measured using ImageJ software. Livers were removed and halved, with one half weighed and digested for $5 \mathrm{~h}$ with $5 \% \mathrm{KOH}$ at $37^{\circ} \mathrm{C}$ with shaking. Schistosome eggs from digested livers were concentrated by centrifugation at $1000 \times \mathrm{g}$ for $10 \mathrm{~min}$ and re-suspended in $1 \mathrm{~mL}$ of $10 \%$ formalin. The number of eggs in a $5 \mu \mathrm{L}$ aliquot was counted in triplicate and the number of eggs per gram (EPG) of the liver was calculated. Small intestines were removed and cleaned of debris before being weighed and digested as per the liver halves. Eggs were also similarly concentrated and counted to calculate the intestinal EPG. 


\subsection{Egg Viability Assays}

The other half of each liver was pooled according to the group, homogenized in $\mathrm{H}_{2} \mathrm{O}$, and placed in identical foil-covered volumetric flasks under bright light to hatch eggs released from the livers. After $1 \mathrm{~h}$, the number of miracidia in $10 \times 50 \mu \mathrm{L}$ aliquots of $\mathrm{H}_{2} \mathrm{O}$ (sampled from the extreme top of each flask) were counted. The number of eggs in each flask at the start of the hatching experiment was determined by liver EPG calculations, allowing the egg hatching index of each group to be calculated by expressing the hatched eggs (miracidia) as a percentage of the total eggs [13].

\subsection{Glucose Consumption and Glycogen Storage Assays}

Five pairs of freshly perfused worms from each vaccinated group were cultured in DMEM $(1000 \mathrm{mg} / \mathrm{L}$ glucose). Media $(50 \mu \mathrm{L})$ from each experiment was collected after $24 \mathrm{~h}$, and the amount of glucose was quantified using a colorimetric glucose assay kit (Sigma), according to the manufacturer's instructions. Glucose levels were expressed relative to media collected from worms recovered from PBS-treated mice (negative control). To measure the glycogen content of these worms, Triton $\mathrm{X}-100$-soluble extracts of each group of five pairs of worms (made by homogenizing the parasites in $1 \%$ Triton $\mathrm{X}-100,40 \mathrm{mM}$ Tris- $\mathrm{HCl}, \mathrm{pH} 7.4$, mixing overnight at $4{ }^{\circ} \mathrm{C}$, and collecting the supernatant by centrifugation at $15,000 \times g$ for $1 \mathrm{~h}$ at $4{ }^{\circ} \mathrm{C}$ ) were assayed for glycogen in a modified procedure described by Gomez-Lechon et al. [19]. Briefly, $0.2 \mathrm{M}$ sodium acetate, $\mathrm{pH} 4.8$, was added to $30 \mu \mathrm{g}$ parasite extract and $50 \mu \mathrm{L}$ glucoamylase $(10 \mathrm{U} / \mathrm{mL})$ to make a reaction volume of $150 \mu \mathrm{L}$. The mixture was incubated at $40{ }^{\circ} \mathrm{C}$ for $2 \mathrm{~h}$ with shaking at $100 \mathrm{rpm}, 40 \mu \mathrm{L}$ was added to a new microplate with $10 \mu \mathrm{L} 0.25 \mathrm{M} \mathrm{NaOH}$, and the amount of glucose was quantified using the colorimetric glucose assay kit. Only worms from trial 1 were available for these experiments. Extracts were made from triplicate sets of parasites and assays were performed three times. Data are presented as the average of each triplicate biological and technical experiment \pm SEM.

\subsection{Experiments Involving Sera from Vaccinated Mice}

Sera were collected from all mice in each group before cercarial challenge and at necropsy. Serum anti-SmChE IgG titers were measured by ELISA against plated SmChEs using standard methods. The cutoff value for each dilution was established as three times the mean OD of the naïve sera for that dilution and the endpoint was defined as the highest dilution above the cutoff value.

To assess whether vaccination-induced antibodies would interact with host serum AChE or BChE, the AChE and BChE activity of pre-challenge sera from all vaccinated and control mice was measured by the Ellman assay. Briefly, $1 \mu \mathrm{L}$ of pre-challenge serum from each mouse was added to $200 \mu \mathrm{L}$ assay buffer (0.1M sodium phosphate, pH 7.4, $2 \mathrm{mM}$ acetylthiocholine [AcSCh], or $2 \mathrm{mM}$ butyrylthiocholine

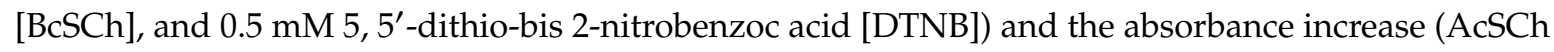
conversion or BcSCh conversion) was monitored over $20 \mathrm{~min}$ at $405 \mathrm{~nm}$ in a Polarstar Omega microplate reader (BMG Labtech). Data are presented as the average of three technical replicates \pm SEM.

\subsection{Statistical analyses}

Statistical differences for all experiments in this chapter were calculated by the Student's $t$ test using GraphPad Prism 7 software. Results are expressed as the mean \pm standard error of the mean (SEM).

\section{Results}

\subsection{Anti-SmChE Polyclonal Antibodies Block Enzyme Activity and Decrease the Viability of Larval S. mansoni in Vitro}

To determine the ability of anti-SmChE-specific polyclonal antibodies to inhibit ChE activity in S. mansoni, and the effect this had on parasite viability, we studied the effects of paralog-specific antibodies on schistosomula at two different timepoints. Treating schistosomula with anti-SmAChE1 IgG, anti-SmAChE2 IgG, or a cocktail of all three anti-SmChE IgGs caused a significant inhibition ( $p \leq$ 
0.01 ) of AChE activity of 56.2\%, 57.1\%, and 59.74\%, respectively, $2 \mathrm{~h}$ after treatment, in comparison with the naïve IgG control (Figure 1A). When schistosomula were incubated with anti-SmBChE1 IgG or a cocktail of all three anti-SmChE IgGs for $2 \mathrm{~h}$, the BChE activity was inhibited by $37.4 \%(p \leq 0.01)$ and $49.3 \%$ ( $p \leq 0.001)$, respectively, compared to the control (Figure 1B). Schistosomula viability was not significantly affected at this timepoint (Figure 1C). Extending the treatment with anti-SmAChE1 IgG, anti-SmAChE2 IgG, or a cocktail of all three anti-SmChE IgGs for $14 \mathrm{~h}$ significantly decreased ( $p \leq 0.001$ ) the AChE activity by $66.9 \%, 70.5 \%$, and $72.6 \%$, respectively, compared to the control (Figure 1D). Similarly, when schistosomula were incubated with anti-SmBChE1 IgG or a cocktail of all three anti-SmChE IgGs for $14 \mathrm{~h}$, the BChE activity decreased significantly ( $p \leq 0.01$ ) by $26.5 \%$ and $35.6 \%$, respectively, compared to the control (Figure 1E). Schistosomula viability was significantly decreased $(p \leq 0.01)$ by all treatments at this timepoint, with the biggest decrease seen in the anti-SmChE cocktail IgG-treated group (Figure 1F).

(A)

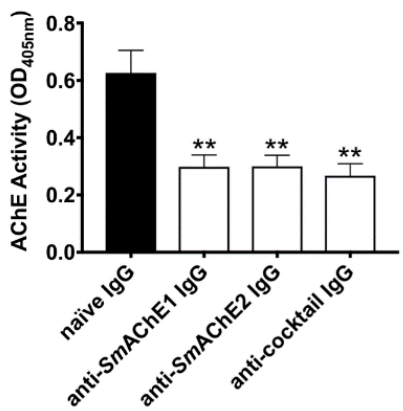

(D)

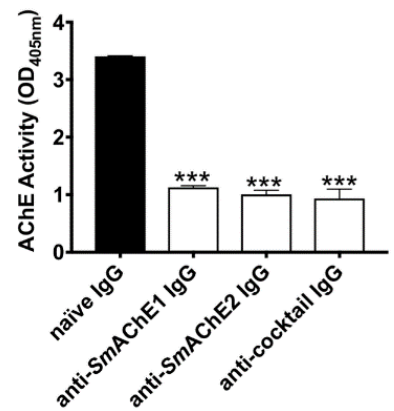

(B)

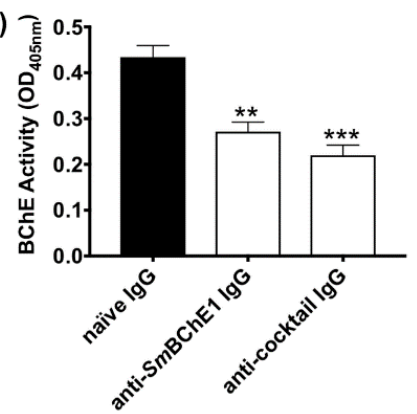

(E)

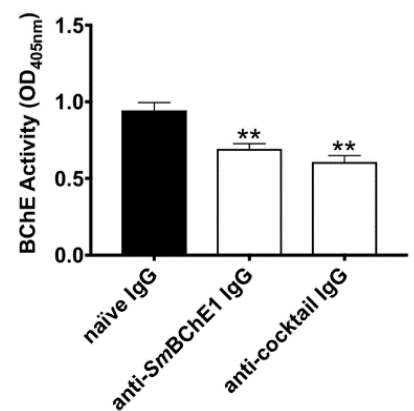

(C)

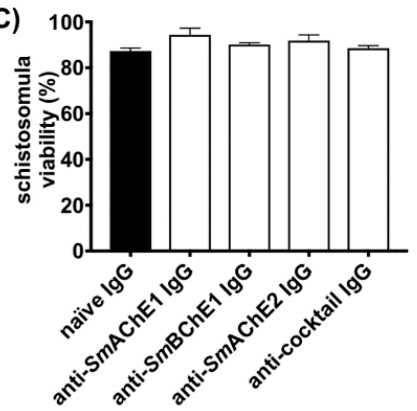

(F)

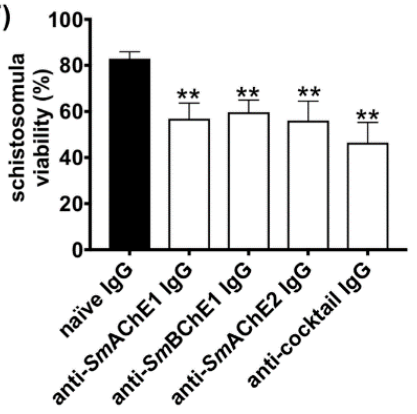

Figure 1. Anti-schistosome cholinesterase ( $\mathrm{S} m \mathrm{ChE}$ ) antibodies inhibit $\mathrm{ChE}$ activity in schistosomula, which leads to a decreased parasite viability. Newly transformed schistosomula (1000/treatment) were incubated in Dulbecco's Modified Eagle Medium (DMEM) in the presence of anti-SmChE IgG and incubated at $37{ }^{\circ} \mathrm{C}$ in $5 \% \mathrm{CO}_{2}$. Naïve IgG served as a negative control. (A) AChE activity $2 \mathrm{~h}$ after treatment. (B) BChE activity $2 \mathrm{~h}$ after treatment. (C) Schistosomula viability $2 \mathrm{~h}$ after treatment. (D) AChE activity $14 \mathrm{~h}$ after treatment. (E) BChE activity $14 \mathrm{~h}$ after treatment. (F) Schistosomula viability $14 \mathrm{~h}$ after treatment. Data represents the mean \pm SEM of two biological and three technical replicates. Significance (relative to the naïve IgG control) determined by the student's $t$ test, where ${ }^{* *} p$ $\leq 0.01$ and ${ }^{* * *} p \leq 0.001$.

\subsection{Effects of Anti-SmChE Antibodies on Adult Worms}

The effects of anti-SmChE antibodies on S. mansoni adult worms was also tested. Freshly perfused worms cultured in the presence of anti-SmAChE1, anti-SmAChE3, or a cocktail of all three anti-SmChEs, showed no significant inhibition of AChE or BChE activity at $2 \mathrm{~h}$ post-treatment, compared to controls. After $24 \mathrm{~h}$ treatment, however, all anti-SmChE IgG-treated groups showed a significant inhibition of AChE and BChE activity, with the anti-SmChE cocktail IgG-treated group displaying the greatest inhibition of AChE activity (Figure 2A,B). The rate of glucose uptake over $24 \mathrm{~h}$ was also measured at this timepoint and all antibody treatments significantly reduced the glucose uptake in adult worms, compared with naïve IgG-treated controls, again with the anti-SmChE cocktail IgG-treated group 
displaying the greatest inhibition (Figure 2C). To determine if anti-SmChE antibodies can play a role in killing adult worms, the antibody experiment was repeated with ten pairs of adult worms per treatment and worm viability post-treatment was assessed. Consistent with inhibition of AChE activity and glucose uptake, the cocktail of anti-SmChE antibodies was the most effective at killing (all worms dead at day 7 post-treatment), compared to controls (Figure 2D).

(A)

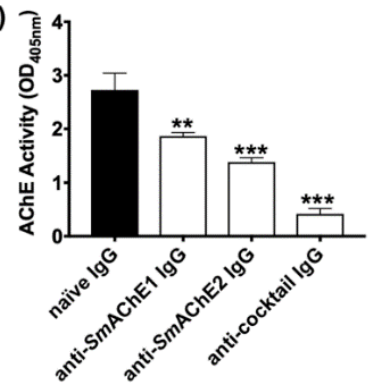

(B)

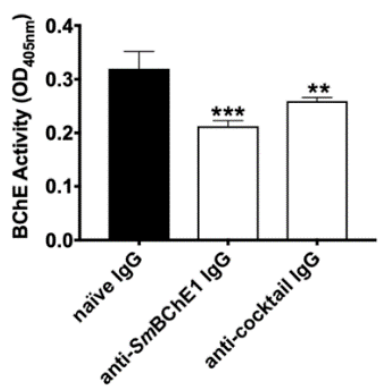

(C)

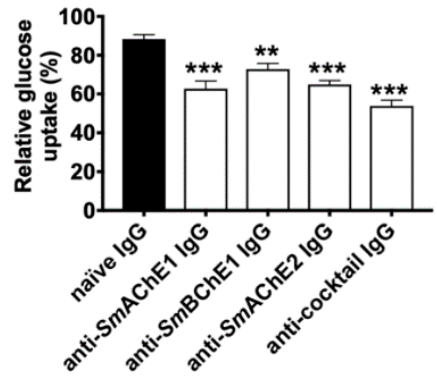

(D)

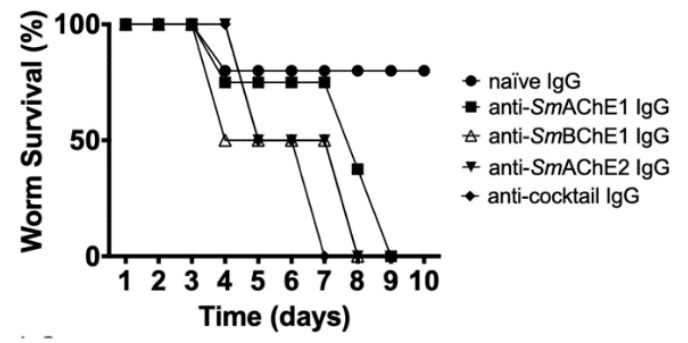

Figure 2. Effects of anti-SmChE antibodies on adult Schistosoma mansoni worms. Ten pairs of freshly perfused worms were incubated in the presence of anti-SmChE purified IgG in DMEM at $37^{\circ} \mathrm{C}$ and $5 \%$ $\mathrm{CO}_{2}$. Naïve IgG served as a negative control. (A) AChE activity $24 \mathrm{~h}$ after treatment. (B) BChE activity $24 \mathrm{~h}$ after treatment. (C) Glucose uptake over $24 \mathrm{~h}$, one day after treatment. (D) Survivability up to 10 days after treatment. The results are the mean \pm SEM of two biological and three technical replicates (A-C) or two biological replicates (D). Significance (relative to the naïve IgG control) determined by the student's $t$ test, where ${ }^{*} p \leq 0.05,{ }^{* *} p \leq 0.01$, and ${ }^{* * *} p \leq 0.001$.

\subsection{Antibody Responses to SmChEs during the Course of Infection and Following PZQ Treatment in Mice}

Antibody responses to all $S m C h E s$ were significantly higher in infected mice than before infection and increased as infection progressed (Figure 3A). In a separate experiment, all anti-SmChE IgG responses were shown to significantly increase $(p \leq 0.001)$ after PZQ treatment (Figure 3B).

(A)

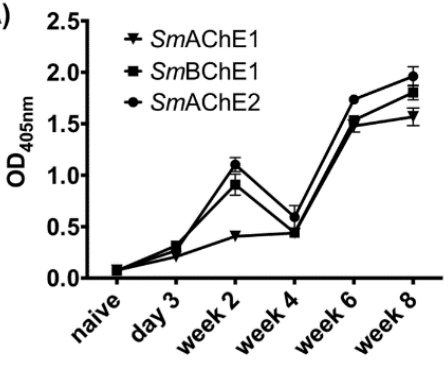

(B)

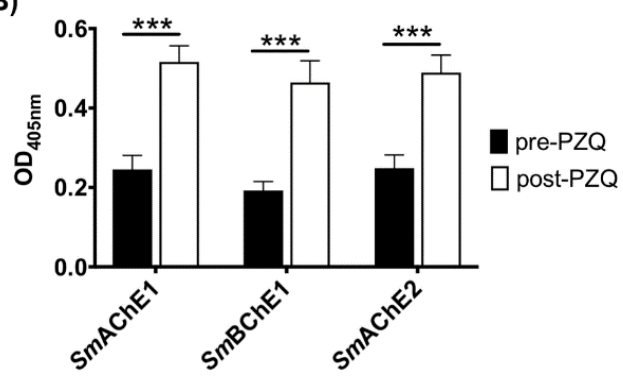

Figure 3. Antibody responses to $S m C h E s$ during the course of infection and following praziquantel (PZQ) treatment in mice. ELISAs showing anti-SmChE IgG responses in mice (A) from 3 days to 8 weeks post-infection $(n=5)$ and $(\mathbf{B})$ before ( 5 weeks post-infection) and 2 weeks after PZQ treatment $(n$ $=11$ ). Data represents the mean of two technical replicates and significance was determined by the student's $t$ test, where ${ }^{* * *} p \leq 0.001$. 


\subsection{Vaccine Efficacy of Recombinant SmChEs in a Mouse Model of Schistosomiasis}

In both vaccine trials, all four groups of mice immunized with $S m C h E s$, either in isolation or as a combination, showed a significant decrease in worm burden (28-39\%), compared to controls, with the $\mathrm{SmChE}$ cocktail-vaccinated group displaying the highest reduction in trial 1 and 2 of $39 \%$ ( $p \leq$ 0.0009 ) and 38\% ( $p \leq 0.0001)$, respectively (Figure 4A,B). Compared to controls, significant decreases in liver egg burdens (expressed as eggs per gram-EPG) were observed for all groups across both trials (13-46\%), except for the SmAChE1-vaccinated group in trial 1. When averaged over both trials, liver egg burdens in the cocktail-vaccinated group showed the greatest reduction (Figure 4C,D). Intestinal egg burdens (expressed as EPG-only determined for trial 2) were significantly reduced for all vaccinated groups, compared to controls, with the greatest reduction seen in the group vaccinated with the $S m \mathrm{ChE}$ cocktail (33\%, $p \leq 0.001)$. (Figure 4E). Egg viability (only assessed for trial 2), as determined by egg hatching from liver homogenates, was significantly reduced in the groups vaccinated with the $S m C h E$ cocktail $(40 \%, p \leq 0.01)$ and SmAChE2 $(46 \%, p \leq 0.01)$ (Figure 4F). While there was no significant reduction in glucose uptake for worms from any of the vaccinated groups, compared to controls, the glycogen content of worms from all vaccinated groups was significantly lower $(24-52 \%, p \leq 0.001)$ than worms from the control group (Figure 5A). A significant reduction in worm length (30\%-50\%) was also observed between worms from all vaccinated groups compared to worms from the control group (Figure 5B). As with the parasitology burden data, worms from the cocktail-vaccinated group showed the greatest decrease in glycogen content and body length. The glucose uptake, glycogen content, and worm size were not significantly different between control and vaccinated groups in trial 2. Serum AChE and BChE activity was also not significantly different between control and vaccinated mice (only measured for trial 2) (Figure S1A,B).

(A)
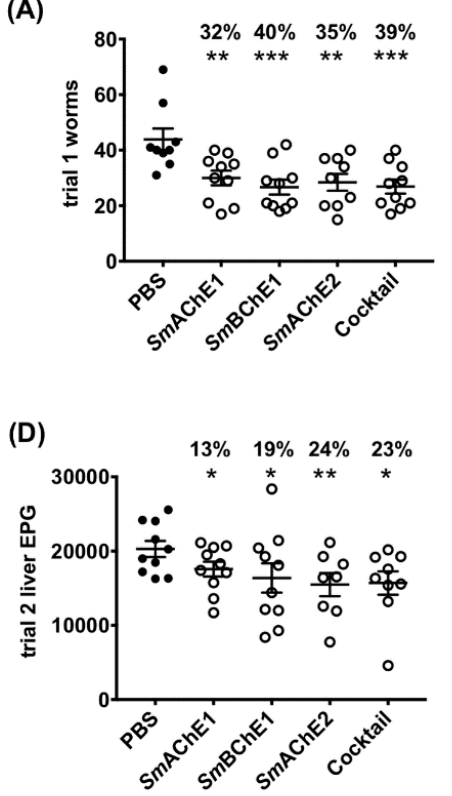

(B)
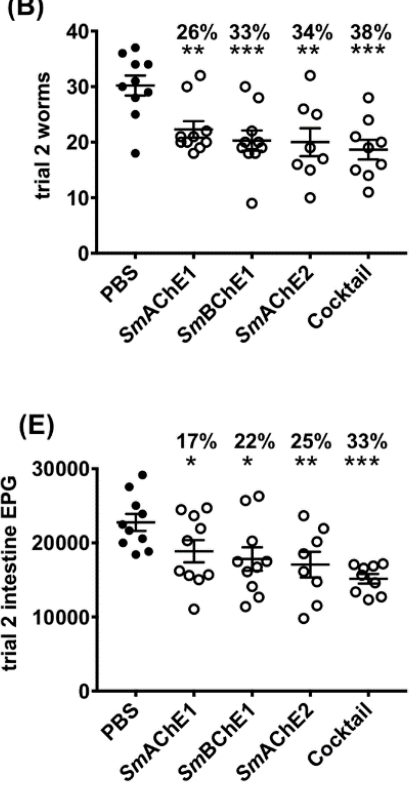

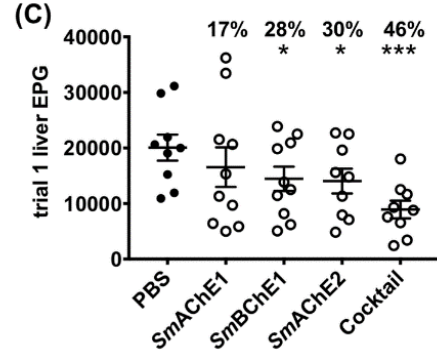

(F)

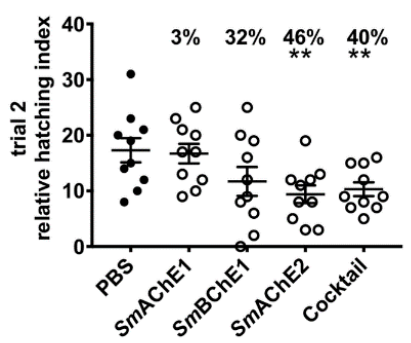

Figure 4. Vaccine efficacy of recombinant $S m \mathrm{ChEs}$ in a mouse model of schistosomiasis. Graphs show parasitology burdens from vaccinated and control mice. (A) Trial 1 adult worms. (B) Trial 2 adult worms. (C) Trial 1 liver eggs per gram (EPG). (D) Trial 2 liver EPG. (E) Trial 2 intestinal EPG. (F) Hatching viability of eggs obtained from the pooled livers of control and vaccinated mice from trial 2. Data are the average of ten replicate counts \pm SEM of hatched miracidia. Significance and percent reductions (if any) for all parameters are measured relative to the control group. Significance determined by the student's $t$ test, where ${ }^{*} p \leq 0.05,{ }^{* *} p \leq 0.01,{ }^{* * *} p \leq 0.001$. 
(A)

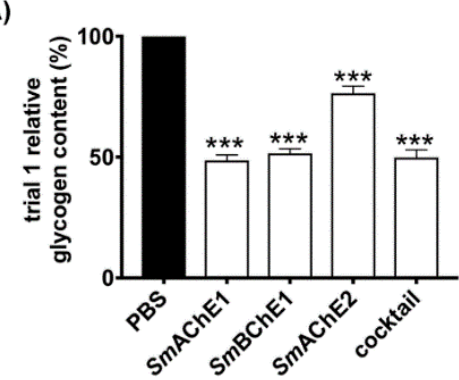

(B)

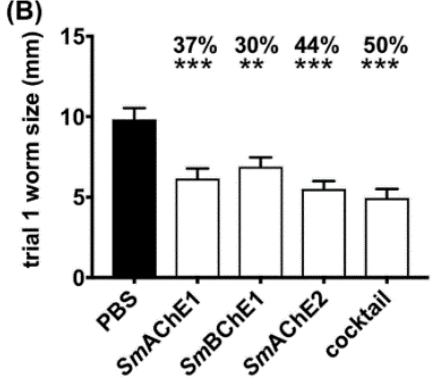

Figure 5. Effect of $S m \mathrm{ChE}$ vaccination on glycogen storage in, and the size of, S. mansoni adult worms. (A) Triton-X-100 extracts were made from five pairs of worms freshly perfused from each vaccinated or control group and the glycogen content in these extracts was measured. Plotted data are the average of triplicate biological and technical experiments \pm SEM. (B) Worm sizes $(\mathrm{mm})$ were assessed by randomly selecting and measuring (ImageJ) at least 20 worms from each group. Significance and percent reductions (if any) for both parameters were measured relative to the control group. Differences for both experiments were measured by the student's $t$ test, where ${ }^{* *} p \leq 0.01$ and ${ }^{* * *} p \leq$ 0.001 .

\subsection{Antibody Responses in Vaccinated Mice}

Moderate to high $(>1,000,000)$ anti-SmChE endpoint titers were seen in the pre-challenge serum of all mice in all vaccinated groups. Post-challenge titers in all groups were four- to ten-fold lower than pre-challenge titers (Tables S1 and S2). There was no correlation between pre-challenge titers and worm burdens in any groups.

\section{Discussion}

Surface-exposed proteins and secreted proteins are effective targets for vaccine development in schistosomes due to their capacity for interaction with host antibodies [20,21]. In this regard, SmChEs are promising candidates, as we have shown in previous immunolocalization studies of $S$. mansoni that SmAChE1, SmBChE1, and SmAChE2 are expressed in the tegument of adult worms and schistosomula and proteomic analysis of $S$. mansoni excretory/secretory (ES) products has confirmed the presence of SmAChE1 and SmBChE1 [8]. Furthermore, RNAi-mediated silencing of all three SmChE genes, both individually and in combination, significantly decreased the schistosomula viability in vitro and parasite survival in vivo [8], implying that these genes are essential for proper worm development and function. Moreover, recent protein array studies have demonstrated high levels of circulating antibodies to $S m B C h E 1$ in individuals exhibiting drug-induced resistance and a low pathology reaction to schistosomiasis, implicating these antibodies in a protective anti-schistosomal response [10,11].

Given that $S m \mathrm{ChEs}$ are accessible to antibody attack and are enzymatically functional [8], catalytic activity can be used to measure the effectiveness of antibody binding as the interaction between enzymes and their corresponding antibodies generally leads to a complete or partial reduction in their enzymatic activity [22-24]. The data presented herein show that antibodies against recombinant SmChEs are capable of inhibiting surface (and, in the case of SmAChE1 and SmBChE1, secreted) enzymatic activities in both schistosomula and adult worms, which is similar to previous studies that have used antibodies raised against parasite-derived AChE to inhibit AChE activity on intact S. mansoni [25], Necator americanus [26], Dictyocaulus viviparus [27], and Electrophorus electricus [28]. The inhibitory effect of antibodies is potentially due to steric hindrance, potentially blocking substrate access to the peripheral anionic site or catalytic gorge of AChE. Indeed, previous studies on rabbit [29], human [30], and bovine [31] AChE have documented the AChE-inhibitory ability of antibodies raised against epitopes other than AChE-active sites.

Consistent with the effects of RNAi-mediated smche gene silencing [8], antibody-mediated SmChE inhibition resulted in a significant decrease in parasite viability. We posit that this mode of enzyme 
inhibition was the cause of eventual parasite death as schistosomula were still viable, compared to controls, at $2 \mathrm{~h}$ after antibody treatment, despite a significant decrease in ChE activity; it was not until after a much longer exposure $(14 \mathrm{~h})$ to anti-SmChE antibodies that the parasite viability was significantly lower.

Glucose uptake in adult worms was also significantly reduced by anti-SmChE antibody treatment. The cholinergic action of surface AChE has been implicated in mediation of the glucose scavenging mechanism in schistosomes [12], AChE-inhibitory metal complexes reduce glucose import in the parasites [13], and we and others have shown that RNAi-mediated silencing of schistosome che genes lessens the uptake of glucose by these parasites [8,14], so it is possible that antibody-mediated impairment of AChE involvement in the glucose uptake pathway is the cause of this effect. It may be that there is some redundancy in the cholinergic functioning of these molecules (even BChEs, like $S m B C h E 1$, can perform a cholinergic role in situations of AChE deficiency [32]), so collective inhibition of the molecules is required to produce a functional deficit. Additionally, given the multiple proposed functions for parasite ChEs [6,33], it is possible that the neutralization of multiple enzymatic targets more profoundly interrupts varied processes of parasite biology than just cholinergic transmission.

Given the relative cytotoxic potential of antibodies against all three SmChEs, as opposed to any single $S m C h E$, we decided to test the efficacy of this antigen cocktail as a vaccine in a mouse model of schistosomiasis. The vaccine efficacy of each individual SmChE was also tested to investigate the relative anti-parasitic effects of each $S m \mathrm{ChE}$ over the cocktail or one another.

Mice vaccinated with the cocktail of $S m \mathrm{ChE}$ antigens displayed the highest level of protection against experimental schistosomiasis, showing the greatest reductions in every parameter tested. An additive protective effect was not readily apparent, however, as protection levels were not significantly different from groups vaccinated with single antigens. Of the groups vaccinated with individual SmAChEs, the SmAChE2-vaccinated group engendered the highest levels of protection. Similar results were reported in a test on the vaccine efficacy of a recombinant AChE from Schistosoma japonicum [14]. Furthermore, vaccine trials using purified secretory AChE from the nematodes Trichostrongylus colubriformis and Dictyocaulus viviparous have resulted in significant protection in animal models [34,35]. It should be noted that schistosomes and other helminth parasites have complex life cycles involving more than one host, so rely on passage of their eggs from the definitive host into the environment to continue their life cycle and transmit disease. Accordingly, mathematical modeling supports the view that a vaccine inducing even partial protection would decrease the parasite egg load in the environment, contributing to the reduction of schistosome infections and interrupting transmission in endemic areas $[1,36]$.

Egg burdens did not concomitantly decrease with worm burdens, but there were significant reductions in egg viability in all but the SmAChE1-vaccinated group, which is an observation we have previously reported when testing the in vivo anti-schistosomal efficacy of AChE-inhibitory drugs [13]. Studies in rats and honey bees have observed abdominal spasms and involuntary muscle contractions when AChE inhibitors have been administered to these organisms [37,38], so a possible explanation for this "less than expected" decrease in egg number but significant reduction in viability could be that ova are being prematurely released as a result of antibody-mediated AChE inhibition affecting reproductive tract motility. It could also be that $\mathrm{ChE}$ vaccination affected the fecundity and egg maturity given the significantly smaller size of worms recovered from vaccinated groups, compared to controls. Indeed, previous studies on insects demonstrated that the suppression of AChE expression considerably reduced the weight and length of surviving organisms [39-41] and severely affected the hatching ability of the eggs laid [39,42]. These reports have suggested that dysregulated cell proliferation and apoptosis during larval growth may be reasons for such phenotypic effects attributed to the absence of AChE, although such a link in trematodes remains to be established. Finally, parasites recovered from vaccinated mice had significantly depleted glycogen stores. Reduced glycogen content and glucose uptake have been previously observed in worms treated with AChE-inhibitory drugs in vitro [13] and attributed to interference with the tegumental AChE-mediated glucose scavenging 
pathway [12] through the inhibition of this enzyme. It could be that the same effect is being orchestrated by the antibody-mediated inhibition of AChE (which would be consistent with the results of in vitro antibody-based experiments), forcing the parasite to rely on its glycogen stores, rather than the scavenging of exogenous glucose, for nutrition.

Even though immunization with SmChEs induced high antibody titers, these antibody levels were not sustained during the course of infection, with titers at necropsy dropping between four- and ten-fold from pre-challenge levels. This would seem to indicate that the specific antibody response induced by immunization was not augmented by natural infection, which is a hypothesis corroborated by the generation of modest anti-SmChE titers during the course of parasite infection in a separate experiment. That being said, the SmChEs used in this study were still capable of inducing moderate levels of protection in the face of modest antibody titers. Treatment with PZQ has been shown to induce antibody-mediated resistance to schistosomiasis in humans through the exposure of parasite antigens to the immune system (and subsequent generation of an antibody response) as a result of tegument damage [43] and protein array studies conducted by us have shown that antibodies to SmBChE1 are significantly upregulated in resistant individuals [10]. The upregulation of $S m C h E$ immune responses following PZQ treatment has been verified in this study. Given that an effective anti-schistosomal vaccine strategy would ideally be linked with chemotherapy [10], it is possible that the vaccine efficacy of antigens such as the ones described here could be increased by vaccination after PZQ treatment due to the augmentation of an already upregulated immune response. The current World Health Organisation (WHO) objective regarding schistosomiasis is to eliminate disease morbidity and mortality as a public health problem-defined by reaching a prevalence of $\leq 5 \%$ and $\leq 1 \%$ of heavy-intensity infections, respectively, in school-age children-in the coming decade [44]. Vaccine-linked chemotherapy has been deemed one the most effective control measures for combating the disease, with this strategy combining the mainstay of schistosomiasis intervention (mass administration of PZQ) with the more long-term benefits of vaccination, and recent modeling has predicted that chemotherapy linked with a vaccine that has an effective duration of protection is the best intervention strategy for achievement of the WHO's goals to significantly reduce the burden of disease caused by schistosomiasis [44].

Supplementary Materials: The following are available online at http://www.mdpi.com/2076-393X/8/2/162/s1: Figure S1: ChE activity in the serum of control and vaccinated mice (trial 2); Table S1: Anti-SmChE IgG titers and worm burdens of vaccinated groups from trial 1; Table S2: Anti-SmChE IgG titers and worm burdens of vaccinated groups from trial 1.

Author Contributions: Conceptualization, B.A.T., A.L. and M.S.P.; methodology, B.A.T. and M.S.P.; validation, B.A.T.; formal analysis, A.L. and M.S.P.; investigation, B.A.T., D.P., L.B. and M.S.P.; writing—original draft, B.A.T. and M.S.P.; writing-review and editing, A.L. and M.S.P.; supervision, A.L. and M.S.P.; funding acquisition, A.L. All authors have read and agreed to the published version of the manuscript.

Funding: This work was funded by NHMRC program grant APP1037304. A.L. was funded by an NHMRC Senior Principal Research Fellowship (AP1117504). B.A.T. was funded by a James Cook University Postgraduate Scholarship.

Acknowledgments: The authors gratefully acknowledge the NIAID Schistosomiasis Research Center of the Biomedical Research Institute, Rockville, MD, USA, for the provision of S. mansoni-infected B. glabrata snails for this work through NIH-NIAID contract HHSN2722017000141 for distribution through BEI resources.

Conflicts of Interest: The authors declare no conflicts of interest. The funders had no role in the design of the study; in the collection, analyses, or interpretation of data; in the writing of the manuscript; or in the decision to publish the results.

\section{References}

1. McManus, D.P.; Dunne, D.W.; Sacko, M.; Utzinger, J.; Vennervald, B.J.; Zhou, X.N. Schistosomiasis. Nat. Rev. Dis. Primers 2018, 4, 13. [CrossRef] [PubMed]

2. Gryseels, B. Schistosomiasis. Infect. Dis Clin. North. Am. 2012, 26, 383-397. [CrossRef] [PubMed]

3. Tebeje, B.M.; Harvie, M.; You, H.; Loukas, A.; McManus, D.P. Schistosomiasis vaccines: Where do we stand? Parasit Vectors 2016, 9, 528. [CrossRef] [PubMed] 
4. Mduluza, T.; Ndhlovu, P.; Madziwa, T.; Midzi, N.; Zinyama, R.; Turner, C.; Chandiwana, S.; Nyazema, N.; Hagan, P. The impact of repeated treatment with praziquantel of schistosomiasis in children under six years of age living in an endemic area for Schistosoma haematobium infection. Memórias Inst. Oswaldo Cruz 2001, 96, 157-164. [CrossRef] [PubMed]

5. Crellen, T.; Walker, M.; Lamberton, P.H.; Kabatereine, N.B.; Tukahebwa, E.M.; Cotton, J.A.; Webster, J.P. Reduced efficacy of praziquantel against Schistosoma mansoni is associated with multiple rounds of mass drug administration. Clin. Infect. Dis. 2016, 63, 1151-1159. [PubMed]

6. Arnon, R.; Silman, I.; Tarrab-Hazdai, R. Acetylcholinesterase of Schistosoma mansoni-Functional correlates-Contributed in honor of Professor Hans Neurath's 90th birthday. Protein Sci. 1999, 8, 2553-2561. [CrossRef]

7. Espinoza, B.; Tarrab-Hazdai, R.; Himmeloch, S.; Arnon, R. Acetylcholinesterase from Schistosoma mansoni: Immunological characterization. Immunol. Lett. 1991, 28, 167-174. [CrossRef]

8. Tedla, B.A.; Sotillo, J.; Pickering, D.; Eichenberger, R.M.; Ryan, S.; Becker, L.; Loukas, A.; Pearson, M.S. Novel cholinesterase paralogs of Schistosoma mansoni have perceived roles in cholinergic signalling and drug detoxification and are essential for parasite survival. PLoS Pathog. 2019, 15, e1008213. [CrossRef]

9. Arnon, R.; Espinoza-Ortega, B.; Tarrab-Hazdai, R. Acetylcholinesterase of Schistosoma mansoni: An antigen of functional implications. Memórias Inst. Oswaldo Cruz 1987, 82, 163-170. [CrossRef]

10. Pearson, M.S.; Becker, L.; Driguez, P.; Young, N.D.; Gaze, S.; Mendes, T.; Li, X.H.; Doolan, D.L.; Midzi, N.; Mduluza, T.; et al. Of monkeys and men: Immunomic profiling of sera from humans and non-human primates resistant to schistosomiasis reveals novel potential vaccine candidates. Front. Immunol. 2015, 6, 213. [CrossRef]

11. Driguez, P.; Li, Y.; Gaze, S.; Pearson, M.S.; Nakajima, R.; Trieu, A.; Doolan, D.L.; Felgner, P.L.; Hou, X.; Cardoso, F.C.; et al. Antibody signatures reflect different disease pathologies in patients with schistosomiasis due to Schistosoma japonicum. J. Infect. Dis. 2016, 213, 122-130. [CrossRef] [PubMed]

12. Camacho, M.; Agnew, A. Schistosoma: Rate of glucose import is altered by acetylcholine interaction with tegumental acetylcholine receptors and acetylcholinesterase. Exp. Parasitol. 1995, 81, 584-591. [CrossRef] [PubMed]

13. Sundaraneedi, M.K.; Tedla, B.A.; Eichenberger, R.M.; Becker, L.; Pickering, D.; Smout, M.J.; Rajan, S.; Wangchuk, P.; Keene, F.R.; Loukas, A.; et al. Polypyridylruthenium(II) complexes exert anti-schistosome activity and inhibit parasite acetylcholinesterases. PLoS Negl. Trop. Dis. 2017, 11, e0006134. [CrossRef]

14. You, H.; Liu, C.; Du, X.; Nawaratna, S.; Rivera, V.; Harvie, M.; Jones, M.; McManus, D. Suppression of Schistosoma japonicum acetylcholinesterase affects parasite growth and development. Int. J. Mol. Sci. 2018, 19, 2426. [CrossRef] [PubMed]

15. Ramalho-Pinto, F.J.; Gazzinelli, G.; Howells, R.E.; Mota-Santos, T.A.; Figueiredo, E.A.; Pellegrino, J. Schistosoma mansoni: Defined system for stepwise transformation of cercaria to schistosomule in vitro. Exp. Parasitol. 1974, 36, 360-372. [CrossRef]

16. Lewis, F.A.; Stirewalt, M.A.; Souza, C.P.; Gazzinelli, G. Large-scale laboratory maintenance of Schistosoma mansoni, with observations on three schistosome/snail host combinations. J. Parasitol. 1986, 72, 813-829. [CrossRef]

17. Wangchuk, P.; Pearson, M.S.; Giacomin, P.R.; Becker, L.; Sotillo, J.; Pickering, D.; Smout, M.J.; Loukas, A. Compounds derived from the Bhutanese Daisy, Ajania nubigena, demonstrate dual anthelmintic activity against Schistosoma mansoni and Trichuris muris. PLoS Negl. Trop. Dis. 2016, 10, e0004908. [CrossRef]

18. Tucker, M.S.; Karunaratne, L.B.; Lewis, F.A.; Frietas, T.C.; Liang, Y.-S. Schistosomiasis. In Current Protocols in Immunology; Coico, R., Ed.; John Wiley and Sons, Inc.: Hoboken, NJ, USA, 2013; pp. 19.1.1-19.1.57.

19. Gómez-Lechón, M.J.; Ponsoda, X.; Castell, J.V. A microassay for measuring glycogen in 96-well-cultured cells. Anal. Biochem. 1996, 236, 296-301. [CrossRef]

20. Mulvenna, J.; Moertel, L.; Jones, M.K.; Nawaratna, S.; Lovas, E.M.; Gobert, G.N.; Colgrave, M.; Jones, A.; Loukas, A.; McManus, D.P. Exposed proteins of the Schistosoma japonicum tegument. Int. J. Parasitol. 2010, 40, 543-554. [CrossRef]

21. Sotillo, J.; Pearson, M.; Becker, L.; Mulvenna, J.; Loukas, A. A quantitative proteomic analysis of the tegumental proteins from Schistosoma mansoni schistosomula reveals novel potential therapeutic targets. Int. J. Parasitol. 2015, 45, 505-516. [CrossRef]

22. Cinader, B.; Suzuki, T.; Pelichová, H. Enzyme-Activation by Antibody. J. Immunol. 1971, 106, 1381. [PubMed] 
23. Arnon, R. Chapter 2-Immunochemistry of Enzymes. In The Antigens; Sela, M., Ed.; Academic Press: Cambridge, MA, USA, 1973; pp. 87-159.

24. Arnon, R. Enzyme inhibition by antibodies. Acta Endocrinologica. Supplementum (Copenhagen) 1975, 194, 133-153. [CrossRef] [PubMed]

25. Espinoza, B.; Parizade, M.; Ortega, E.; Tarrab-Hazdai, R.; Zilberg, D.; Arnon, R. Monoclonal antibodies against acetylcholinesterase of Schistosoma mansoni: Production and characterization. Hybridoma 1995, 14, 577-586. [CrossRef] [PubMed]

26. Pritchard, D.I.; Leggett, K.V.; Rogan, M.T.; McKean, P.G.; Brown, A. Necator americanus secretory acetylcholinesterase and its purification from excretory-secretory products by affinity chromatography. Parasite Immunol. 1991, 13, 187-199. [CrossRef]

27. McKeand, J.B.; Knox, D.P.; Duncan, J.L.; Kennedy, M.W. The immunogenicity of the acetylcholinesterases of the cattle lungworm Dictyocaulus viviparus. Int. J. Parasitol. 1994, 24, 501-510. [CrossRef]

28. Williams, R.M. Antibodies to acetylcholinesterase. Proce. Natl. Acad. Sci. USA 1969, 62, 1175-1180. [CrossRef]

29. Brimijoin, S.; Mintz, K.P.; Prendergast, F.G. An inhibitory monoclonal antibody to rabbit brain acetylcholinesterase. Studies on interaction with the enzyme. Mol. Pharmacol. 1985, 28, 539-545.

30. Sorensen, K.; Brodbeck, U.; Rasmussen, A.G.; Norgaard-Pedersen, B. An inhibitory monoclonal antibody to human acetylcholinesterases. Biochim. Biophys. Acta 1987, 912, 56-62. [CrossRef]

31. Wolfe, A.D. The monoclonal antibody AE-2 modulates fetal bovine serum acetylcholinesterase substrate hydrolysis. Biochim. Biophys. Acta 1989, 997, 232-235. [CrossRef]

32. Boudinot, E.; Taysse, L.; Daulon, S.; Chatonnet, A.; Champagnat, J.; Foutz, A.S. Effects of acetylcholinesterase and butyrylcholinesterase inhibition on breathing in mice adapted or not to reduced acetylcholinesterase. Pharmacol. Biochem. Behav. 2005, 80, 53-61. [CrossRef]

33. Day, T.A.; Chen, G.Z.; Miller, C.; Tian, M.; Bennett, J.L.; Pax, R.A. Cholinergic inhibition of muscle fibres isolated from Schistosoma mansoni (Trematoda:Digenea). Parasitology 1996, 113, 55-61. [CrossRef] [PubMed]

34. Griffiths, G.; Pritchard, D.I. Vaccination against gastrointestinal nematodes of sheep using purified secretory acetylcholinesterase from Trichostrongylus colubriformis-an initial pilot study. Parasite Immunol. 1994, 16, 507-510. [CrossRef] [PubMed]

35. McKeand, J.B.; Knox, D.P.; Duncan, J.L.; Kennedy, M.W. Immunisation of guinea pigs against Dictyocaulus viviparus using adult ES products enriched for acetylcholinesterases. Int. J. Parasitol. 1995, 25, 829-837. [CrossRef]

36. Alsallaq, R.A.; Gurarie, D.; Ndeffo Mbah, M.; Galvani, A.; King, C. Quantitative assessment of the impact of partially protective anti-schistosomiasis vaccines. PLoS Negl. Trop. Dis. 2017, 11, e0005544. [CrossRef]

37. Jarvie, E.M.; Cellek, S.; Sanger, G.J. Potentiation by cholinesterase inhibitors of cholinergic activity in rat isolated stomach and colon. Pharmacol. Res. 2008, 58, 297-301. [CrossRef]

38. Williamson, S.; Moffat, C.; Gomersall, M.; Saranzewa, N.; Connolly, C.; Wright, G. Exposure to Acetylcholinesterase Inhibitors Alters the Physiology and Motor Function of Honeybees. Front. Physiol. 2013, 4. [CrossRef]

39. Kumar, M.; Gupta, G.P.; Rajam, M.V. Silencing of acetylcholinesterase gene of Helicoverpa armigera by siRNA affects larval growth and its life cycle. J. Insect Physiol. 2009, 55, 273-278. [CrossRef]

40. Hui, X.-M.; Yang, L.-W.; He, G.-L.; Yang, Q.-P.; Han, Z.-J.; Li, F. RNA interference of ace1 and ace2 in Chilo suppressalis reveals their different contributions to motor ability and larval growth. Insect Mol. Biol. 2011, 20, 507-518. [CrossRef]

41. Ye, X.; Yang, L.; Stanley, D.; Li, F.; Fang, Q. Two Bombyx mori acetylcholinesterase genes influence motor control and development in different ways. Sci. Rep. 2017, 7, 4985. [CrossRef]

42. Xiao, D.; Lu, Y.H.; Shang, Q.L.; Song, D.L.; Gao, X.W. Gene silencing of two acetylcholinesterases reveals their cholinergic and non-cholinergic functions in Rhopalosiphum padi and Sitobion avenae. Pest. Manag. Sci. 2015, 71, 523-530. [CrossRef] 
43. Harder, A.; Andrews, P.; Thomas, H. Praziquantel: Mode of action. Biochem. Soc. Trans. 1987, 15, 68. [CrossRef] [PubMed]

44. Kura, K.; Truscott, J.; Toor, J.; Anderson, R.M. Modelling the impact of a Schistosoma mansoni vaccine and mass drug administration to achieve morbidity control and transmission elimination. PLoS Negl. Trop. Dis. 2019, 13, e0007349. [CrossRef] [PubMed] 